\title{
Inheritance and Development of Chinese Dialect Culture
}

\author{
Hongmei Wang \\ School of Culture and Media \\ Chongqing University of Arts and Sciences \\ Chongqing, China 402160
}

\begin{abstract}
Chinese dialects not only build the richness and diversity of modern Chinese, but also carry rich and diverse regional cultures, and are also important witnesses and records of the ancient Chinese culture. With the continuous promotion of mandarin and the increasingly close communication among regions, the inheritance of Chinese dialect culture is facing an embarrassing situation, and its development has also shown a declining trend. It can be said that the inheritance and development of Chinese dialect culture has been greatly challenged. In this context, this article analyzes the current status of cultural heritage and development of Chinese dialects from the perspective of presenting the functional value of Chinese dialects, and proposes strategies for the cultural heritage and development of Chinese dialects from multiple aspects.
\end{abstract}

Keywords-dialect; Chinese dialect culture; dilemma; development strategy

\section{INTRODUCTION}

From the perspective of sociolinguistics, Chinese dialect refers to a language that is different from mandarin. In the vast land of China, there are many Chinese dialects, mainly including official dialects, $\mathrm{Wu}$ dialects, Hunan dialects, Jiangxi dialects, Hakka dialects, Guangdong and Fujian dialects. In some regions with complex dialects, there are many local dialects. The differences among Chinese dialects, mandarin and Chinese dialects are mainly in terms of phonetics, grammar, and vocabulary. In the course of the development of Chinese language, it has always been accompanied by the inheritance and development of dialects. As early as the Oracle era, the northern and southern dialects had been recorded. Lu Xun pointed out in the study of historical classics that the difficulty of reading classics was evidence of dialect writing. [1] In order to facilitate the smooth and convenient communication between people in different regions, "mandarin" has been promoted in all ages, and contemporary China is also vigorously promoting mandarin. However, this does not negate the unique functions of the dialects and the significance of its heritage and development. Chinese dialects not only build the richness and diversity of modern Chinese, but also carry rich and diverse regional cultures. They are also important witnesses and records of the long-standing Chinese national culture. Dialects are used in a large number of classics, poems, novels and plays. There are a lot of dialects in "The
Book of Songs" and "The Songs of Chu". In addition, dialects also play a special function of connecting emotions Some commentators have said that hometown dialect is the most beautiful nostalgia.

In the 1950s, China began to promote mandarin. In the 1980s, "promoting mandarin that is universally used in the country" was written into the Constitution. At the beginning of the new century, the "Law of the People's Republic of China on Spoken and Written Languages" was promulgated which established mandarin as the common language of China from a legal perspective. With the continuous promotion of mandarin and the increasingly close communication among regions, the inheritance of Chinese dialect culture is facing an embarrassing situation, and its development has also shown a declining trend. In other words, the inheritance and development of Chinese dialect culture has been greatly challenged. The current research on the protection, inheritance and development of Chinese dialect culture mainly includes the following aspects: the first is from the perspective of language policy; the second is from the perspective of language ecology; the third is from the perspective of intangible cultural heritage. These studies show that scholars have paid attention to and inherited the dialect culture, and also enriched the theoretical basis for the development of Chinese dialect culture. On the basis of presenting the functional value of Chinese dialects, this article analyzes the current status of cultural inheritance and development of Chinese dialects, and proposes strategies for cultural inheritance and development of Chinese dialects from multiple aspects.

\section{ANALYSIS ON THE STATUS QUO OF CULTURAL} INHERITANCE AND DEVELOPMENT OF CHINESE DIALECTS

There are different opinions on the division of Chinese dialects. At present, the divisions recognized by most researchers are the seven major dialect areas mentioned above. Mandarin dialects are northern dialects, which are the basic dialects of mandarin and represented by Beijing dialect. $\mathrm{Wu}$ dialect is also known as Jiangnan dialect, represented by Shanghai dialect and Suzhou dialect, is mainly used in Shanghai, Zhejiang, southern Jiangsu, southern Anhui, eastern Jiangxi and other places. Hunan dialect is represented by Changsha dialect (new Hunan dialect) and Hengyang dialect (old Hunan dialect), and there are many differences 
between them. Jiangxi dialects are represented by Nanchang dialect and Fuzhou dialect, which are mainly used in Jiangxi, eastern Hunan, southwestern Anhui, and southeastern Hubei. Hakka dialects are represented by Meizhou dialect, and the phonology is similar to Jiangxi dialects. And it is widely used in eastern Guangdong, western Fujian, southeastern Guangxi, Taiwan and other southern regions of China. Guangdong dialect is also known as Cantonese, is used in central and western Guangdong, eastern Guangxi, and Hong Kong and Macao. It is one of the most complex Chinese dialects. Fujian dialect is mainly used in Fujian, Hainan, eastern Guangdong and other places, with obvious internal branches. The phonology represented by Quanzhou dialect, Fuzhou dialect, and Puxian dialect, northern Fujian dialect, mid Fujian dialect is closest to the ancient Chinese phonology. [2]

As early as the 1980s, China began to vigorously promote mandarin. In recent years, with the decline of the development of Chinese dialects, especially the local dialects in the seven major dialect areas, the state and local governments have begun to pay attention to the inheritance and development of Chinese dialect culture. Some provisions for the use of dialects have been added to the text usage method, calling on the public to protect and inherit dialects and promote the development of dialect culture while promoting and using mandarin. Nonetheless, the inheritance and development of Chinese dialect culture is still not optimistic, mainly in the following aspects. First, the use of dialects has gradually decreased; the state has vigorously promoted mandarin; and it has required official activities and news media to use mandarin for publicity. The mainstream value orientation of the use of mandarin has been formed within the society, making people in many regions gradually use mandarin, and less use dialects for communication, and reducing the use of dialects. The foundation of cultural inheritance of the dialect is gradually shaken. Second, the diversity of dialects is gradually decreasing. For example, Hakka dialects face the predicament of inheritance during the migration of Hakka dialect users. What is more serious is that local dialects, especially small language family dialects with counties and townships as units, are blocked from inheriting. The disappearance of some dialects directly leads to the decrease of dialect diversity. When the symbol of regional culture, historical culture and national culture of a certain region disappear, it means the absence and fault of culture. Third, the cultural value of dialects has not been well tapped and played. Reduction in the number of people who follow and use dialects leads to a decline in dialect usage and diversity. When people rarely use and no longer value dialects, dialect culture naturally has "no successor", and the culture carried by the dialect is gradually drowned in the history. [3] There are many reasons for the dilemma of cultural heritage and development of Chinese dialects. From the perspective of social orientation, the state vigorously promotes mandarin; mandarin is also used in formal occasions and official media; but it lacks the protection and inheritance mechanism for the dialect. It has not given dialects sufficient status from the legal level or the mainstream value-oriented level of the society. From the perspective of pragmatic values, with the rapid development of society and economy, the exchanges between regions are becoming closer and more convenient. The universal value of mandarin has shown great advantages, while dialects have gradually lost their pragmatic value. From the perspective of individual ideological understanding, with the influence of social values, more and more young people, especially young people who leave their hometown, are proud to use mandarin, and are unwilling or even ashamed to use dialects that express regional characteristics. The disconnection of dialect inheritance brings great crisis to the development of dialect culture.

\section{RESEARCH ON STRATEGIES FOR CULTURAL INHERITANCE AND DEVELOPMENT OF CHINESE DIALECTS}

There is an old slang tongue of "eastern Qi using wild language and southern using slang". Since ancient times, Chinese dialects have always been an important cultural carrier rooted in regions, history, and nations. Although dialects are not the mainstream standard language used by the government and recognized by the public due to their inherent limitations, they bear cultural marks that "mandarin" does not possess. Only by protecting Chinese dialects and carrying out the inheritance and development of dialect culture from the perspective of macro policies and implementation strategies, can the Chinese language continue to develop in a diversified language environment and the 5,000-year-old culture of the Chinese nation be more fully sustained and inherited. [4]

As mentioned above, as early as the 1980s, the state established mandarin as the common language of China from the legal level. The "Constitution", the "Education Law of the People's Republic of China", the "Law of the People's Republic of China on Spoken and Written Languages" and other laws and regulations all stipulate relevant measures to promote mandarin. In contrast, the inheritance and development of dialect culture lacks legal guarantees and institutional norms. In the "Opinions on Strengthening the Protection of China's Intangible Cultural Heritage" and "Chinese Language Resources Protection Project", it is clear that dialect culture should be protected and inherited as intangible cultural heritage and important language resources. However, China has not yet formulated specific measures on protecting dialects, inheriting and developing dialect culture from the legal level. At present, while promoting mandarin, the state must increase the importance of dialect culture, formulate relevant protection measures at the macro policy level, and provide a legal basis for the inheritance and development of dialect culture. The seven major dialect areas, in accordance with the laws and regulations formulated by the state, should develop local systems to protect the dialect and inherit the dialect culture in accordance with local conditions, so that the inheritance and development of the dialect culture can be based on evidence and rules.

In the process of dialect culture inheritance and development, all sectors of society must assume corresponding responsibilities, and take multiple measures to implement the policies and calls for the protection of dialect and the inheritance of culture. First, under the overall guidance of the local government, a non-governmental 
organization that inherits and develops dialect culture will be established, and relevant management methods and effective measures will be formulated to collect and record the dialects in the region, so as to carry out excavation and inheritance of dialect culture. Dialect culture gets better inheritance and development in the benign interaction between government guidance and non-governmental organization operation. The second is to use modern information technology to scientifically protect the dialect culture. With the beneficial experience of the audio database of the Chinese Language Resource Protection Project, it can rely on libraries, colleges and universities, and folk dialect protection and inheritance institutions, and establish a dialect database. By means of written records, voice records and video records, dialects can be recorded and protected in a more three-dimensional way, providing a solid resource base and sufficient materials for the use of dialects, the inheritance of dialects and the continuous exploration of the unique value of dialect culture. [5] Third, local media should increase the promotion of local dialects. While promoting mandarin, newspapers, magazines, radio, websites and other media can provide a certain space for the use and inheritance of dialects, so as to arouse the enthusiasm of dialect users to use dialects in informal communication, and change the one-sided value judgment of abandoning dialects and rejecting dialects. Relying on the power of mass media, it highlights the unique value of dialect and the significance of inheriting and developing dialect culture. The fourth is to carry out "bilingual teaching" of mandarin and dialects properly. On the basis of ensuring the promotion of local dialect culture on campus, elective courses of local dialect culture should be set up appropriately, and regional cultural knowledge and historical cultural knowledge carried by local dialect should be taught in dialect, so as to guide students to be good inheritors of dialect culture while speaking mandarin. The fifth is to use modern media methods to fully mobilize dialect users to use dialects and protect the dialect. As a communication tool, dialects must have new values on the basis of continuous use. The inheritance of dialect culture also depends on the promotion of dialect usage. It is convenient to use Weibo, WeChat and other popular media methods in contemporary society to build a communication platform for dialect users and inheritors, such as establishing WeChat groups and public accounts of local dialects, publishing related content such as dialect voice, word meaning, and inheriting dialect culture, holding folklore-related activities, etc., and using convenient "online" communication method. Then, it can better achieve cross-regional communication of dialect culture. From the "online" groups spontaneously formed by dialect users and inheritors, it can gradually explore and establish a multiparty interactive and diversified network platform to attract professional guidance from universities and research institutions and the injection of commercial capital. In the process of protecting dialect, inheriting and developing dialect culture, it is necessary to give play to the strong synergy of mass participation.

\section{CONCLUSION}

Dialects are not only a communication tool in a certain area, but also an important mark of regional culture, historical culture, and national culture, and even an important component in the process of inheritance and development of the Chinese national culture. With the rapid economic and social development and increasingly prosperous cultural exchanges, no matter from the level of the national macro policy or the specific implementation strategy of all sectors of society, it is still a long way to explore the inheritance and development of dialect culture. It is important to find the balance among promoting mandarin, protecting dialect and inheriting and developing dialect culture, which requires people to constantly explore and work together.

\section{REFERENCES}

[1] Zhang Sumin. Study on Collision and Coexistence of Dialects and Mandarin from the Perspective of Dialect News [J]. Journal of Wenzhou University (Natural Science Edition), 2011, 32 (4): 53. (in Chinese)

[2] Cao Zhiyun. Endangered dialects, cultural fragments and the mission of dialect scholars [J]. Journal of Chinese Linguistics, 2014 (7): 212. (in Chinese)

[3] Wu Yonghuan. On Significance and Strategies of Protecting the Cultural Heritage of Chinese Dialects [J]. Journal of Renmin University of China, 2008 (4): 42. (in Chinese)

[4] Wang Saisai. Properly Handling the Relationship between Mandarin and Dialects [J]. Youth Literator, 2013 (30): 121. (in Chinese)

[5] Wang Shuying. On the Value and Protection Strategy of Chinese Dialects [J]. Southeast Academic Research, 2017 (4): 238. (in Chinese) 